\title{
About the Theory of Tree Embedding
}

\author{
Alexandre Boudet and Hubert Comon \\ LRI, CNRS URA 410 \\ Université Paris-Sud, Centre d'Orsay \\ 91405 Orsay Cedex, France
}

\begin{abstract}
We show that the positive existential fragment of the theory of tree embedding is decidable.
\end{abstract}

\section{Introduction}

Symbolic Constraints, i.e. formulae interpreted in some term structure, have been revealed to be extremely useful in logic programming and theorem proving. Among such constraints, the ordering constraints can be used in expressing ordered strategies at the formula level instead of the inference level. This allows to cut further the search space, while keeping the completeness of the strategy [7]. Solving ordering constraints also allows for a nice lifting of orderings from the ground level to the terms with variables: define $s>t$ by $\forall \vec{x} . s>t$ where $\vec{x}$, the variables of $s, t$, range over all ground terms. This provides with more powerful orderings for termination proofs in rewriting theory.

Up to now, the satisfiability of ordering constraints has been studied for some orderings on terms: Venkataraman showed that the existential fragment of the theory of the subterm ordering is decidable, while the $\Sigma_{3}$ fragment is undecidable [10]. These results have been extended recently to infinite trees [9]. Comon showed that the existential fragment of the theory of any total lexicographic path ordering is decidable [1]. This result has been extended to any recursive path ordering over a total precedence by Jouannaud and Okada [6]. On the other hand, the $\Sigma_{4}$ fragment of the theory of any partial recursive path ordering is undecidable (provided that the signature is rich enough) [8].

All these works have left some open questions, among which the decidability of the existential theory of a partial recursive path ordering. Among the partial recursive path orderings, the tree embedding is the simplest one: the precedence is assumed to be empty. Actually the tree embedding is contained in all (partial) recursive path orderings. We do not solve here the decidability problem in its full generality, but we hope to contribute to the general solution: we show that the positive existential fragment of the theory of tree embedding is decidable.

The proof is carried out by elementary techniques which are quite different from those in $[10,9,1,6]$. Indeed, for subterm problems, $[10,9]$ use some "test sets" showing that, if there is a solution, there is some solution which has a "small" size. They also use normal forms of inequations systems in which all inequations $s \geq t$ have a variable on the left. As we will see, it is not possible to follow this technique with the embedding relation. On the other hand, $[1,6,7]$ use 
the linearity of the ordering in many places: the main problem is the expression of the successor function on the term level. Of course, we cannot use such a technique with the embedding which is not a linear ordering.

First, we set up precisely the problem in section 2 . Then we give some obvious transformation rules in section 3 . Using some stability properties of the set of solutions, we derive some additional rules reported in section 4 . In section 5 , we introduce more syntactic constructions in order to express easily strategies and we solve the problem of multiple upper bounds. We give section 6 the last rule which allows to break non-trivial cycles which necessarily occur in problems that are irreducible w.r.t. the other rules. Then we show that the whole set of rules terminates, thus leading to the decidability result.

\section{Syntax and Semantics}

Terms are built on the finite (ranked) alphabet $F$ of function symbols and a set of variable symbols $X$. The resulting algebra is denoted $T(F, X)$ (Or $T(F)$ when $X$ is empty). We use mainly the notations of [3]. For example, the result of replacing a term $t$ with a term $u$ at position $p$ in $s$ is denoted $s[u]_{p}$. This notation is also used in order to indicate that $u$ occurs at position $p$ in $s$. The root position (empty string) is denoted by $\Lambda$.

The tree homeomorphic embedding (or simply embedding) is the reduction relation associated with the rewrite system consisting of all rules

$$
f\left(x_{1}, \ldots, x_{n}\right) \rightarrow x_{i}
$$

for $i \in\{1, \ldots, n\}$ and $f \in F\left(x_{1}, \ldots, x_{n}\right.$ are variables $)$. Embedding is a well ordering on terms [4]; it will be denoted $\unlhd$ (more precisely, $s \unlhd t$ if $t \rightarrow s$ using the above rules).

More operationally, we can use the following definition:

$$
\begin{aligned}
& s \equiv f\left(s_{1}, \ldots, s_{n}\right) \unlhd g\left(t_{1}, \ldots, t_{m}\right) \equiv t \quad \text { iff } \\
& \quad \exists i, s \unlhd t_{i} \\
& \text { or } f=g \text { and } \forall i, s_{i} \unlhd t_{i}
\end{aligned}
$$

Inequational formulae are disjunctions of existentially quantified conjunctions of either inequations $s \leq t$ or equations $s=t$ where $s, t \in T(F, X)$. The set of variables of an inequational formula $I$ is denoted by $\operatorname{Var}(I)$. $T$ and $\perp$ respectively denote the trivial and the unsatisfiable inequational problem.

$I$ is interpreted as follows: a ground assignment $\sigma$ (i.e. a mapping associating each variable of $I$ with a term in $T(F)$ ) satisfies $s \leq t$ if $s \sigma \unlhd t \sigma$. Similarly, it satisfies $s=t$ if $s \sigma \equiv t \sigma$ (三 is the identity of terms). This interpretation extends to inequational formulae in the usual way. If all solutions of an inequational formula $I$ are also solutions of an inequational formula $J$, we will write $I \vDash J$.

The problem we address here is the satisfiability of inequational problems. "Most" of the problems are satisfiable. Let us show some examples of unsatisfiable problems in increasing complexity. In all examples, $f, h$ are binary function symbols, $g, k$ are unary function symbols and $a, b$ are constants. 
Example 1 .

$g(y) \leq x \wedge g(x) \leq y$ is not satisfiable since we can deduce $g(g(y)) \leq y$, which is unsatisfiable. This illustrates the monotonicity of embedding. We also used the fact that embedding contains the subterm ordering.

Example 2.

$g(y) \leq x \wedge x \leq f(y, g(g(a))) \wedge b \leq f(x, y)$ is not satisfiable since, from the first two inequations, we can conclude that $g(y)$ must be embedded in $g(g(a))$. It follows that $y$ is either $a$ or $g(a)$. Next, $x$ must be embedded in $f(g(a), g(g(a)))$. Then $b \leq f(x, y)$ cannot be satisfied. This illustrates the fact that only finitely many terms are embedded in a given term.

Example 3.

$x \leq g(k(y)) \wedge x \leq k(g(y)) \wedge k(y) \leq x \wedge g(y) \leq x$ is not satisfiable. Indeed, either $x=g\left(x_{1}\right)$, or $x=k\left(x_{1}\right)$ for some $x_{1}$, or the top symbol of $x$ is neither $g$ nor $k$.

- In the first case, we deduce from the second inequation that $g\left(x_{1}\right) \leq g(y)$, hence $x_{1} \leq y$. From the third inequation, we deduce that $k(y) \leq g\left(x_{1}\right)$, hence $k(y) \leq x_{1}$. Now there is a contradiction: $x_{1} \leq y \wedge k(y) \leq x_{1}$ is unsatisfiable as in example 1 .

- The second case $\left(x=k\left(x_{1}\right)\right)$ is symmetric of the first one.

- In the last case (the top symbol of $x$ is neither $g$ nor $k$ ), we can deduce from the first inequation that $x \leq y$ which, together with $k(y) \leq x$ leads to a contradiction.

This example illustrates the problem of "multiple upper bounds": if some term $t$ is embedded in both $g(u)$ and $h(v)$, then it must be embedded in either $u$ or $v$.

\section{A first set of transformation rules}

The technique we will use for deciding the satisfiability of inequational formulae is now classical (see $[2,5]$ ): it consists of rewriting the formula, using some rules which preserve the satisfiability, until the problem becomes trivially decidable.

Our first set of rules is quite straightforward to derive. It is displayed in figure 1 . Let us call $\mathcal{R}_{0}$ this set of rules. All formulae are assumed to be hept in disjunctive normal form. Then the rules of figure 1 transform an inequational formula into an inequational formula.

Lemma 1. All rules in $\mathcal{R}_{0}$ preserve the set of solutions of inequational formulae. Moreover, $\mathcal{R}_{0}$ is terminating and irreducible inequational formulae are disjunctions of conjunctions of equations and inequations whose at least one member is a variable.

Actually, after one normalization w.r.t. $\mathcal{R}_{0}$, equations become useless. Discarding them preserves the satisfiability. Hence, for sake of simplicity, we assume now that inequational formulae do not contain any equation. We can assume this property along all other transformations. Also, every disjunction can be treated separately (there is no interaction between them). For this reason, we will sometimes forget that inequational problems contain disjunctions. 
Embedding Rules (Emb)

$$
\begin{aligned}
& f\left(s_{1}, \ldots, s_{n}\right) \leq g\left(t_{1}, \ldots, t_{m}\right) \rightarrow \bigvee_{i=1}^{m} f\left(s_{1}, \ldots, s_{n}\right) \leq t_{i} \\
& \quad \text { if } f \neq g \\
& f\left(s_{1}, \ldots, s_{n}\right) \leq f\left(t_{1}, \ldots, t_{n}\right) \rightarrow\left(\bigwedge_{i=1}^{n} s_{i} \leq t_{i}\right) \vee \bigvee_{i=1}^{n} f\left(s_{1}, \ldots, s_{n}\right) \leq t_{i}
\end{aligned}
$$

Unification Rules (Unif)

$$
\begin{aligned}
f\left(s_{1}, \ldots, s_{n}\right)=g\left(t_{1}, \ldots, t_{m}\right) & \rightarrow \perp \\
f\left(s_{1}, \ldots, s_{n}\right)=f\left(t_{1}, \ldots, t_{n}\right) & \rightarrow s_{1}=t_{1} \wedge \ldots \wedge s_{n}=t_{n} \\
x=s \wedge P \rightarrow & x \wedge P\{x \mapsto s\} \\
& \text { if } x \notin \operatorname{Var}(s), x \in \operatorname{Var}(P), \text { and } \\
& \text { if } s \in X, \operatorname{then} s \in \operatorname{Var}(P) \\
& s=s \rightarrow T \\
x=s[x]_{p} \rightarrow \perp & \text { if } p \neq \Lambda
\end{aligned}
$$

Occur Check Rules (C'heck)

$$
\begin{aligned}
& \begin{aligned}
& x \leq t[x] \rightarrow \top \\
& t_{1}\left[x_{1}\right]_{p_{1}} \leq x_{2} \wedge \ldots \wedge t_{n}\left[x_{n}\right]_{p_{n}} \leq x_{1} \rightarrow \perp \text { if } p_{i} \neq \wedge \text { for some } i \\
& x_{1} \leq x_{2} \wedge \ldots \wedge x_{n} \leq x_{1} \wedge P \rightarrow P\left\{x_{2} \mapsto x_{1}, \ldots, x_{n} \mapsto x_{1}\right\} \wedge \bigwedge_{i=2}^{n} x_{i}=x_{1}
\end{aligned}
\end{aligned}
$$

Removing ground terms occurring on the right (Ground 1)

$$
\begin{gathered}
x \leq t \wedge P \rightarrow \bigvee_{u \leq t} x=u \wedge P\{x \mapsto u\} \\
\quad \text { if } t \in T(F)
\end{gathered}
$$

Fig. 1. The set $\mathcal{R}_{0}$ of rules

\section{Basic Properties of Inequational Problems}

In this section, we state the basic (crux) properties of inequational problems.

Lemma 2. The set of solutions of an inequational problem is stable by homomorphism i.e. given any tree-morphism $\theta$ from $T(F)$ into itself if $\sigma \models I$, then $\theta \circ \sigma \models I \theta$.

For example, if there is no constant occurring in $I$, and $a \in F$ is a constant, then the set of solutions is stable under the morphism $\{a \mapsto t\}$ for any ground term $t$.

We can also take advantage of the following straightforward remark: 
Lemma 3. If $I$ is a conjunction of inequations of the form $x \leq t$ where $x$ is a variable and $t$ is a non-ground term, then, for any ground term $u$, the substitution $\sigma_{u}$ mapping every variable of $I$ on $u$ is a solution of $I$.

As a corollary, the following rule preserves the satisfiability:

\section{Separate}

$I_{1} \wedge I_{2} \wedge s_{1} \leq t_{1} \wedge \ldots \wedge s_{n} \leq t_{n} \rightarrow I_{1}$

If $\operatorname{Var}\left(I_{2}\right) \cap\left(\operatorname{Var}\left(s_{1}, \ldots, s_{n}\right) \cup \operatorname{Var}\left(I_{1}\right)\right)=\emptyset$, and for every $i, \operatorname{Var}\left(t_{i}\right) \& \operatorname{Var}\left(I_{1}\right) \cup \operatorname{Var}\left(s_{1}, \ldots, s_{n}\right)$, and

$I_{2}$ consists of inequations $x \leq s$ where $x$ is a variable and $s$ is a non-ground term.

Indeed, if $\sigma$ is a solution of $I_{1}$, then the substitution $\sigma \circ \sigma_{u}$ is a solution of $I_{1} \wedge I_{2} \wedge s_{1} \leq t_{1} \wedge \ldots \wedge s_{n} \leq t_{n}$ for some large enough $u$. Actually, it is sufficient to choose for $u$ a term larger than $s_{1} \sigma, \ldots, s_{n} \sigma$. This is always possible, except if $F$ contains two distinct constants and no function symbol of arity larger or equal to 2. This latter case is discarded now: the case of unary function symbols can be solved by means of automata.

The above remarks also suggest to remove inequations $s \leq x$ from $I$ when $s$ is a ground term. Indeed, if $\sigma$ is a solution of the remainder of the problem, then it will be enough to compose $\sigma$ with a well chosen homomorphism $(\{a \mapsto s\}$ for example), leading to a solution of $I$. This is not completely correct as the remainder of $I$ may contain occurrences of constants (and hence be modified by the morphism). So, we will "freeze" the inequations $s \leq x$ when the variables of $s$ only occur on the left, waiting until the rest of the problem is solved. Then, either $s \leq x$ has become ground along the transformation (leading to either $\mathrm{T}$ or $\perp$ ) or else we will be able to construct a solution out of a solution of the non-frozen part, thanks to the stability by homomorphism. Let us show an example:

\section{Example 4.}

Let $I$ be $g(a) \leq x \wedge x \leq f(a, y) \wedge x \leq f(y, a) \wedge y \leq x$. We freeze $g(a) \leq x$. The two inequations $x \leq f(a, y) \wedge x \leq f(y, a)$ are equivalent to $x \leq f(a, a) \vee x \leq y$ (see the next sections). In the first case, using the $\mathcal{R}_{0}$-normalization, we get a contradiction with the frozen part $g(a) \leq x$ which has become ground: $g(a) \leq$ $f(a, a)$. In the second case, we get $y=x$. From any substitution $\sigma=\{x \mapsto$ $s ; y \mapsto s\}$ (which is a solution of the hot part) we get a solution $\theta \circ \sigma$ of $I$, by composition with the morphism $\theta=\{b \mapsto g(a)\}$ where $b$ is any constant occurring in $s$.

Let us consider a new syntactic construction (for strategic purposes only) in inequational formulae: conjunctions of inequations may be surrounded by brackets \{\} in which case, the inequations are frozen. This will be managed by the following rule: 


\section{Freeze}

$\left\{I_{1}\right\} \wedge s \leq t \wedge I_{2} \rightarrow\left\{I_{1} \wedge s \leq t\right\} \wedge I_{2}$

If there is no variable in $s$ occurring in some right hand side of an inequation of $I_{2}$

The non-frozen part of a conjunction of inequations $I$ will also be called the hot part of $I$ and written $H(I)$.

\section{$5 \quad$ Elimination of multiple upper bounds}

The Separate rule allows to eliminate variables which occur only in right sides of inequations (take $I_{2}=\emptyset$ ). Freeze eliminates (from the hot part) variables which occur only on the left. It is also possible to eliminate the variables which are bound only once; the rule:

\section{Eliminate}

$\{P\} \wedge Q \wedge x \leq s \rightarrow\{P\{x \mapsto s\}\} \wedge Q\{x \mapsto s\}$

If $x$ does not occur ir $s$ nor in any left member of an inequation of $P$ or $Q$

preserves the satisfiability. Indeed, if $\sigma$ is a solution of $\{P\} \wedge Q \wedge x \leq s$, consider the substitution $\theta$ which is identical to $\sigma$, except on $x$ where $x \theta \equiv s \sigma$. Of course, $\theta$ still satisfies any inequation in which $x$ does not occur. It also obviously satisfies $x \leq s$. Then, it only remains to consider inequations in which $x$ occurs on the right. Let $y \leq t$ be such an inequation. Then $y \theta \equiv y \sigma \unlhd t \sigma \unlhd t \theta$ since $x \sigma \unlhd s \sigma \equiv x \theta$. Hence $\theta$ is also a solution of $y \leq t$.

The problem is now illustrated by example 3 : some variable may be bounded twice by "incompatible" terms. In order to express that $x$ is bounded by both $s$ and $t$, we write $x \in s \& t$. This will be useful for keeping track of the deductions we already considered.

Let $\mathcal{E}$ be the set of terms in $T(F \cup\{\&\}, X)$ where $\&$ is assumed to be associative-commutative and is used in infix notation. Ground expressions $e \in \mathcal{E}$ are interpreted as finite sets of terms as follows:

$$
\begin{aligned}
\llbracket s \& t \rrbracket & \stackrel{\text { def }}{=} \llbracket s \rrbracket \cap \llbracket t \rrbracket \\
\llbracket f\left(s_{1}, \ldots, s_{n}\right) \rrbracket & \stackrel{\text { def }}{=} \llbracket s_{1} \rrbracket \cup \ldots \cup \llbracket s_{n} \rrbracket \cup\left\{f\left(t_{1}, \ldots, t_{n}\right) \mid \forall i, t_{i} \in \llbracket s_{i} \rrbracket\right\} \\
\mathcal{I}\left(f\left(s_{1}, \ldots, s_{n}\right)\right) & \stackrel{\text { def }}{=}\left\{f\left(t_{1}, \ldots, t_{n}\right) \mid \forall i, t_{i} \in \mathcal{I}\left(s_{i}\right)\right\} \\
\mathcal{I}(s \& t) & \stackrel{\text { def }}{=} \llbracket s \& t \rrbracket
\end{aligned}
$$

For example, $\mathcal{I}(g(f(a, g(a)) \& h(b, g(a))))=\{g(a), g(g(a))\}$ : the first $g$ cannot be erased, whereas the other occurrences of $g$ (below $\&$ ) can be erased. Let us emphasize that $[\cdot]$ stands for the set of all terms embedded in the expression, 
whereas $\mathcal{I}$, which is only used above the first occurrence of a $\&$, imposes that no symbol is erased until an $\&$ is reached.

Then, we introduce a new predicate symbol $\in$ with the following meaning: the solutions of $s \in e$, where $s \in T(F, X)$ and $e \in \mathcal{E}$ is the set of ground substitutions $\sigma$ such that $s \sigma \in \mathcal{I}(e \sigma)$. For example, the solutions of $x \in g(k(y)) \& k(g(y))$ are the substitutions $\{x \mapsto g(s) ; y \mapsto s\}$ for some ground term $s$ and the substitutions $\{x \mapsto k(s) ; y \mapsto s\}$ for some ground term $s$.

The occur-check relation is the smallest reflexive-transitive relation $\leq_{o c}^{I}$ on the variables of an inequational problem $I$ such that:

- if $u[x] \leq y$ is in $I$, then $x \leq_{o c}^{I} y$

- if $x \in y \& e$ is in $I$, then $x \leq_{o c}^{I} y$

Gathering together the bounds of a variable $x$ is expressed using the new predicate symbol:

\section{Bounds}

$x \leq s_{1} \wedge \ldots \wedge x \leq s_{n} \rightarrow x \in s_{1} \& \ldots \& s_{n}$

if $x$ is maximal w.r.t. $\leq_{o c}$ among the variables occurring in inequations of the hot part of the problem, and $s_{1}, \ldots, s_{n}$ are all the terms occurring on the right of an inequation whose left member is $x$, and $n \geq 2$.

The correctness of this rule is quite straightforward. "Deciphering" membership conditions consists in replacing $x \in e$ with a conjunction of inequations; for $e \in \mathcal{E}$, we define $\mathcal{D}(s \in e)$ as the set of inequations $s \leq t$ where $t$ is any normal form of $e$ w.r.t the rewrite system

$$
\begin{aligned}
& u \& v \rightarrow u \\
& u \& v \rightarrow v
\end{aligned}
$$

Now, we are ready to give the additional rules dealing with the new syntactic constructions. They are given in figures 2 and 3 .

The rule system $\mathcal{R}_{1}$ acts on normal forms w.r.t. $\mathcal{R}_{0}$. It consists of all rules of figures 2, 3 and of the rule Bounds. The new rules Separate and Freeze which are displayed in this figure are generalizations of the previous ones (hence we do not have to give new names), incorporating the new syntactic construction.

Lemma 4. All rules of $\mathcal{R}_{1}-\{$ Separate, Unbounded-Var $\}$ preserve the satisfiability of inequational problems.

This is mostly a mechanical verification. Note however that, for $e \in \mathcal{E}$, we always have $\mathcal{I}(e) \subseteq \llbracket e \rrbracket$, but the converse is false when the top symbol of $e$ is not $\&$. Consequently, the Ground 2 rule or the second Freeze rule which replaces $x \in e$ with $\mathcal{D}(x \in e)$ may, locally, add solutions. But they don't add solutions to the original inequational problem as the additional solutions can be found in another disjunct. 


\section{Eliminate}

$\{P\} \wedge Q \wedge x \leq s \rightarrow\{P\{x \mapsto s\}\} \wedge Q\{x \mapsto s\}$

if $x$ does not occur in $s$ nor in any left member of an inequation of $P$ or $Q$

\section{Ground 2}

$x \in e \rightarrow \mathcal{D}(x \in e)$

if there is an inequation $x \leq t$ with $t$ ground in $\mathcal{D}(x \in e)$

\&-normalization

$$
\begin{aligned}
& x \in e\left[f\left(t_{1}, \ldots, t_{n}\right) \& g\left(s_{1}, \ldots, s_{m}\right)\right] \rightarrow \bigvee_{i=1}^{n} x \in e\left[t_{i} \& g\left(s_{1}, \ldots, s_{m}\right)\right] \vee \\
& \bigvee_{i=1}^{m} x \in e\left[f\left(t_{1}, \ldots, t_{n}\right) \& s_{i}\right] \\
& \text { if } f \neq g \\
& x \in e\left[f\left(t_{1}, \ldots, t_{n}\right) \& f\left(s_{1}, \ldots, s_{n}\right)\right] \rightarrow x \in e\left[f\left(s_{1} \& t_{1}, \ldots, s_{n} \& t_{n}\right)\right] \vee \\
& \bigvee^{n} x \in e\left[t_{i} \& f\left(s_{1}, \ldots, s_{n}\right)\right] \vee \\
& i=1 \\
& \bigvee_{i=1}^{n} x \in e\left[f\left(t_{1}, \ldots, t_{n}\right) \& s_{i}\right]
\end{aligned}
$$

\section{Ground 2}

$$
\begin{aligned}
& x \in e \rightarrow \mathcal{D}(x \in e) \\
& \quad \text { if there is an inequation } x \leq t \text { with } t \text { ground in } \mathcal{D}(x \in e)
\end{aligned}
$$

Check-\&

$$
\begin{aligned}
x \in e\left[u[x]_{p} \& s\right] \rightarrow x & \in e[s] \\
& \text { if there is no \& symbol along the path } p
\end{aligned}
$$

Check- $\epsilon$

$$
\begin{aligned}
P \wedge x_{0} \in x_{1} \& e_{1} \wedge \ldots \wedge x_{n} \in x_{0} \& e_{n+1} \rightarrow & x_{0}=x_{1} \wedge \ldots \wedge x_{0}=x_{n} \\
& \wedge x_{0} \in e_{1} \& \ldots \& e_{n+1} \\
& \wedge P\left\{x_{1} \mapsto x_{0} ; \ldots ; x_{n} \mapsto x_{0}\right\}
\end{aligned}
$$

Fig. 2. The set $\mathcal{R}_{1}$ of rules. Part I 
Separate

$$
\bigwedge_{i \in \mathcal{I}} x_{i} \in e_{i} \wedge \bigwedge_{j \in \mathcal{J}} y_{j} \in e_{j}^{\prime} \wedge \bigwedge_{k \in \mathcal{K}} s_{k} \leq t_{k} \wedge P_{-V} \rightarrow \bigwedge_{j \in \mathcal{J}} y_{j} \in e_{j}^{\prime} \wedge P_{-V}
$$

If 1 . no rule from $\mathcal{R}_{0}, \&$-normalization, Ground 2 can be applied to the problem on the left

2. $V$ is a subset of the variables occurring in the problem on the left

3. For all $i \in \mathcal{I}, \operatorname{Var}\left(x_{i}, e_{i}\right) \subseteq V$

4. For all $j \in \mathcal{J}, \operatorname{Var}\left(e_{\jmath}^{\prime}\right) \cap \bar{V} \neq \emptyset$

5. For all $k \in \mathcal{K}, \operatorname{Var}\left(s_{k}\right) \cap V=\emptyset$ and $\operatorname{Var}\left(t_{k}\right) \cap V \neq \emptyset$

6. $\operatorname{Var}\left(P_{-V}\right) \cap V=\emptyset$.

\section{Unbounded-Var}

$$
\begin{gathered}
x \in t\left[u[y]_{p} \& v\right]_{q} \rightarrow x \in t[v]_{q} \\
x \in t[y]_{p} \rightarrow T
\end{gathered}
$$

If $y$ doesn't occur in any left side of a membership condition or an inequation, there is no \& along the path $p$, and the \&-normalization rules cannot be applied.

Freeze

$$
\left\{I_{1}\right\} \wedge s \leq t \wedge I_{2} \rightarrow\left\{I_{1} \wedge s \leq t\right\} \wedge I_{2}
$$

If there is no variable in $s$ which occurs in either some right hand side of an inequation of $I_{2}$ or in some right side of a membership condition of $I_{2}$.

$$
\left\{I_{1}\right\} \wedge x \in e \wedge I_{2} \rightarrow\left\{I_{1} \wedge \mathcal{D}(x \in e)\right\} \wedge I_{2}
$$

If $x$ does not occur in $e$ nor in $I_{2}$.

Fig. 3. The set $\mathcal{R}_{1}$ of rules. Part II

Also, we need an extension of lemma 3 in order to prove the correctness of the Separate rule:

Lemma 5. Assume that $I$ consists of membership conditions $x \in \in$ only and that $I$ is irreducible w.r.t. \&-normalization and Ground 2. Then, for every ground term $t$, there is a solution $\sigma$ of $I$ such that, for every variable $x$ of $I$, $t \unlhd x \sigma$.

Proof. Replace every atom $x \in t_{x}\left[z_{1} \& e_{1}, \ldots, z_{n} \& e_{n}\right]$ in the inequational problem $I$ with $\exists x_{1}, \ldots, x_{n} . x=t_{x}\left[x_{1}, \ldots, x_{n}\right] \wedge x_{1} \in z_{1} \& e_{1} \ldots \wedge x_{n} \in z_{n} \& e_{n}$ where $t_{x}$ doesn't contain any occurrence of $\&$ (this is of course correct). We get an equational part $I_{1}$ and a conjunction $I_{2}$ of atoms of the form $x \in z \& e$ where $z$ is a variable (by irreducibility w.r.t. \&-normalization). Then, for every ground term $u, \sigma_{u}$ is a solution of $I_{2}$. We prove this result by induction on the sum of sizes of all expressions occurring on the right of the membership conditions of $I_{2}$. 
If all membership conditions are of the form $x \in t$ where $t$ does not contain any $\&$, this is a consequence of lemma 3 . In the same way, if the only occurrences of $\&$ are in expressions $z_{1} \& \ldots \& z_{n}$ where $z_{1}, \ldots, z_{n}$ are variables, then $\sigma_{u}$ is obviously a solution.

Now, assume that there is a condition $x \in z_{1} \& \ldots \& z_{n} \& e$ where $e$ is not a variable. Then, by irreducibility w.r.t. \&-normalization, $e$ can be written $u\left[y_{1} \& e_{1}, \ldots, y_{k} \& e_{k}\right](k \geq 0)$ where $u$ does not contain any occurrence of $\&$ and $y_{1}, \ldots, y_{k}$ are variables. Moreover, there is an index $i$ such that Ground 2 would not apply on $x \in y_{i} \& e_{i}$. (For, otherwise, Ground 2 would apply on $\left.x \in z_{1} \& \ldots \& z_{n} \& e\right)$. Applying the induction hypothesis to $I_{2}$ in which $x \in$ $z_{1} \& \ldots \& z_{n} \& e$ is replaced with $x \in y_{i} \& e_{i}$, we get the result that $\sigma_{u}$ is a solution of this new membership system. But, if $x \sigma_{u} \in \llbracket y_{i} \sigma_{u} \& e_{i} \sigma_{u} \rrbracket$, then, a fortiori, $x \sigma_{u} \in \llbracket z_{1} \sigma_{u} \& \ldots \& z_{n} \sigma_{u} \& e \sigma_{u} \rrbracket$.

We conclude the proof by considering the substitutions $\theta_{u}$ which assign to each $x \in \operatorname{Var}(I)$ the term $t_{x}\left[x_{1}, \ldots, x_{n}\right] \sigma_{u}: \theta_{u}$ is a solution of $I$ and $u \unlhd x \theta_{u}$.

Lemma 6. The rules Separate and Unbounded-Var preserve the satisfiability of inequational problems.

Lemma 7. Assume that $\phi$ is $\in$-free and $\phi \underset{\mathcal{R}_{1}}{\stackrel{*}{\longrightarrow}} \psi$. Then all variables occurring as left members of membership conditions in $\psi$ do not occur in any left hand side of an inequation of $\psi$.

This is a consequence of the maximality conditions on $x$ in the Bounds rule.

Lemma 8. Considering $\mathcal{R}_{1}$ as acting on normal forms w.r.t. $\mathcal{R}_{0}$ (i.e. we assume an $\mathcal{R}_{0}$-normalization after each $\mathcal{R}_{1}$-reduction), we get a terminating reduction.

Indeed, if we only consider the hot part of inequational problems, the number of variables occurring in left sides of inequations is not increasing. No rule can introduce variables in left hand sides of inequations, except the rules which replace a variable with some other variable in the whole problem (with the Check and Check- $\epsilon$ rules) and the Ground 2 rule. In the former case, the number of variables occurring in left sides of inequations is not increasing. In the latter case, by $\mathcal{R}_{0}$-normalization, $x$ must be replaced everywhere with a ground term, which means that it does no longer occur in a left side of an inequation. Now, by lemma 7 , the number of variables occurring in left sides of inequations of the hot part is strictly decreasing as soon as Bounds is applied.

Now, if there is no occurrence of Bounds in the reduction sequence, the \&normalization alone terminates (and is independent of the other rules), Ground rules eliminate variables and all other rules reduce the size of the problem.

\section{Eliminating Cycles}

In this section, we restrict our attention to the hot part $\phi$ of an inequational problem $I$ irreducible for $\mathcal{R}_{0} \cup \mathcal{R}_{1}$. The transformation rules that we have given 
so far yield problems whose hot parts are either $T$ or $\perp$, or problems involving some non-trivial cycles as defined below. For studying the properties of such cycles, we associate a weighted, oriented graph $G^{\phi}$ with an inequational problem $\phi$, irreducible for $\mathcal{R}_{0} \cup \mathcal{R}_{1}$. Examining this graph will give us both the application conditions of our last rule and the termination proof.

\subsection{Interpreting Inequational Problems into Graphs}

The vertices of $G^{\phi}$ are the variables of $\phi$. The graph $G^{\phi}$ has two different kind of arcs, and several arcs may connect two vertices:

Definition 9. Let $\phi$ be an inequational problem, irreducible for $\mathcal{R}_{0} \cup \mathcal{R}_{1}$. The graph $G^{\phi}$ has the variables of $\phi$ for vertices and

- There is an $\operatorname{arc} x \stackrel{p}{=} y$ if and only if there is an inequation $u[x]_{p} \leq y$ in $\phi$, where the weight $|p|$ of the arc is the length of position $p$.

- There is an arc $x \stackrel{p}{\stackrel{1}{p}} y$ if and only if there is an atom $x \in e$ in $\phi$, and $x \leq t[y]_{p}$ belongs to $\mathcal{D}(x \in e)$, where the weight $|p|$ of the arc is the length of position $p$.

A non-trivial cycle is a cycle (i.e., a non-trivial path with the same target and origin) involving ares of the form $x \stackrel{p}{=} y$ and arcs of the form $x \stackrel{p}{\longleftarrow} y$. The weight of an arc in superscript will be dropped if irrelevant.

It turns out that if an inequational problem, irreducible for $\mathcal{R}_{0} \cup \mathcal{R}_{1}$ is not Tor $\perp$, then the associated graph $G^{\phi}$ will contain a non-trivial cycle.

It follows from the lemma 7 that if there is an arc $x \stackrel{p_{i}}{\longleftarrow} x_{i}$ in $G^{\phi}$, then all the in-going arcs of $x$ are of the form $x \stackrel{p_{j}}{\longleftarrow} x_{j}$, and they all come from the same atom $x \in e\left[x_{1}, \ldots, x_{n}\right]$ of $\phi$.

In case $G^{\phi}$ contains no arcs of the form $x \stackrel{p}{=} y$, then Separate applies with $V=\operatorname{Var}(\phi)$, turning the hot part of the problem into $T$. We have the following straightforward lemma:

Lemma 10. Let $\phi$ be an inequational problem, irreducible for $\mathcal{R}_{0} \cup \mathcal{R}_{1}$. Then $G^{\phi}$ has at least one arc of the form $x \stackrel{p}{=} y$.

We can now restrict our attention to the problems involving arcs of the form $x \stackrel{p}{=} y$. Now, a crucial property of $G^{\phi}$ will locate where to apply our last rule:

Lemma 11. Let $\phi$ be an inequational problem, irreducible for $\mathcal{R}_{0} \cup \mathcal{R}_{1}$. If $G^{\phi}$ contains an are $x \stackrel{p}{=}$ with $p \neq \Lambda$, then there is a vertex $z \geq_{o c} y$ whose in-going arcs are all of the form $z \stackrel{q}{\longleftarrow} z^{\prime}$ with $|q| \neq 0$.

Proof. Consider a maximal (i.e. without any in-going arcs) strongly connected component $C$ of $G^{\phi}$. Let $\operatorname{Var}(C)$ be the set of vertices of $C$. $C$ cannot be reduced to a singleton $\{x\}$ since Unbounded-Var, Separate, or Eliminate would 
apply. Indeed, in this case, $x$ having no in-going arc could not appear anywhere in a left-hand side of an inequation or a membership condition.

We assume now that $\operatorname{Var}(C)$ is not a singleton, and we first show, by contradiction that $C$ contains an arc of the form $x \stackrel{p}{=} y$ with $p \neq A$. Assume the converse. Then there is no inequation in $\phi$ of the form $u[x]_{p} \leq y$ with $p \neq A$ and $x \in \operatorname{Var}(C)$ because if $y \in \operatorname{Var}(C)$, then the edge $x \stackrel{p}{=} y$ would belong to $C$ and otherwise, $C$ would have an in-going arc. Let $\operatorname{Atom}(C)$ be the set of atoms of $\phi$ containing a variable of $\operatorname{Var}(C)$. The atoms of $\operatorname{Atom}(C)$ are then of one of the following forms:

- $x \in e$, where $x \cup \operatorname{Var}(e) \subseteq \operatorname{Var}(C)$,

- $s \leq x$, where $x \in \operatorname{Var}(C)$ and $\operatorname{Var}(s) \cap \operatorname{Var}(C)=\emptyset$,

- $x \in e$, where $x \notin \operatorname{Var}(C)$ and $\operatorname{Var}(e) \cap \operatorname{Var}(C) \neq \emptyset$.

In this case, $\operatorname{Var}(C)$ matches the application conditions of Separate, a contradiction.

Consider now an $\operatorname{arc} x \stackrel{p}{=} y$ of $C$ and let $z$ be a variable, such that $z \geq_{o c} y, z$ being maximal for the occur-check relation. $C$ having no in-going arc, $z$ belongs to $C$. Since $z$ is maximal for $\geq_{o c}$, its only in-going arcs are of the form $z \stackrel{q}{q} z_{i}^{\prime}$, with $q \neq A$.

The previous lemma shows that one of the following rules can be applied to a problem $\phi$ irreducible for $\mathcal{R}_{0} \cup \mathcal{R}_{1}$, whenever $\phi$ contains an inequation.

$$
\begin{aligned}
& \text { Explode } \\
& x \in t\left[e_{1}, \ldots, e_{n}\right]_{p_{1}, \ldots, p_{n}} \wedge \psi \rightarrow \\
& \quad \exists \vec{z} x=t\left[z_{1}, \ldots, z_{n}\right]_{p_{1}, \ldots, p_{n}} \wedge \bigwedge_{i=1}^{n} z_{i} \in e_{i} \wedge \psi\left\{x \mapsto t\left[z_{1}, \ldots, z_{n}\right]_{p_{1}, \ldots, p_{n}}\right\}
\end{aligned}
$$

if no rule of $\mathcal{R}_{0} \cup \mathcal{R}_{1}$ applies, and $x$ is maximal for $\leq_{o c}$, and there is an inequation $u[y]_{p} \leq x^{\prime}$ with $p \neq A$ in $\psi$, and $x \geq_{o c} x^{\prime}$, and $p_{1} \neq \Lambda, \ldots, p_{n} \neq \Lambda$, where $e_{1}, \ldots, e_{n}$ are the maximal subterms of $t$ with top function symbol \&.

The rule Explode preserves the sets of solutions, according to the semantics of the predicate $\epsilon$. Note in addition that the proof of lemma 11 shows that if $G^{\phi}$ contains an edge $x \stackrel{p}{=} y$ with $p \neq A$, then it contains a non-trivial cycle since both an edge $x \stackrel{p}{\Longleftarrow} y$ and an edge $x^{\prime} \stackrel{q}{\longleftarrow} y^{\prime}$ belong to the same strongly connected component. The partial correctness of our algorithm is given by the following lemma:

Lemma 12. If the hot part $\phi$ of an inequational problem $I \not \equiv \perp$, irreducible for $\mathcal{R}_{0} \cup \mathcal{R}_{1}$, is not $\mathrm{T}$, then the rule Explode applies.

Indeed, such a problem must contain an inequation $u[y]_{p}$ with $p \neq A$, otherwise Separate would apply, reducing the hot part of the problem to T. In this case, lemma 11 shows that Explode applies. 
We are left to show that the process of applying Explode and re-normalizing the problem with $\mathcal{R}_{0} \cup \mathcal{R}_{1}$ terminates.

\subsection{Termination}

We prove the termination by defining a well-founded ordering $>W$ on graphs such that if Explode is applied to a problem $\phi$ and the resulting problem is re-normalized for $\mathcal{R}_{0} \cup \mathcal{R}_{1}$, yielding a problem $\psi$, then $G^{\phi}>_{W} G^{\psi}$.

Definition 13. Let $\operatorname{Path}\left(G^{\phi}\right)$ be the set of maximal paths of $G^{\phi}$ of the form $x \stackrel{p_{1}}{=} y_{1} \stackrel{p_{2}}{\longleftarrow} y_{2} \stackrel{p_{3}}{\longleftarrow} y_{3} \cdots y_{n-1} \stackrel{p_{n}}{\longleftarrow} y_{n}$, which do not contain twice a same arc (by maximal we mean that there is no further arc $y_{n} \stackrel{p_{n+1}}{\longleftarrow} y_{n+1}$ ).

With a path $\mathcal{P}=x \stackrel{p_{1}}{=} y_{1} \stackrel{p_{2}}{\longleftarrow} y_{2} \stackrel{p_{3}}{\longleftarrow} y_{3} \cdots y_{n-1} \stackrel{p_{n}}{\longleftarrow} y_{n} \in \operatorname{Path}\left(G^{\phi}\right)$, we associate the weight $W(\mathcal{P})=\left(\left|p_{1}\right|, n \cdot\left|p_{n}\right| \cdot\left|p_{n-1}\right| \cdots\left|p_{2}\right|\right)$. The ordering $>_{W}$ on graphs is the multiset extension of the ordering obtained by comparing lexicographically the weights of the paths of $\operatorname{Path}\left(G^{\phi}\right)$, using the usual ordering on natural numbers for the first component, and alphabetic ${ }^{1}$ ordering on words of natural numbers for the second component.

Lemma 14. Let $\phi$ be an inequational problem, irreducible for $\mathcal{R}_{0} \cup \mathcal{R}_{1}$. Assume Explode, is applied to $\phi$, followed by a normalization for $\mathcal{R}_{0} \cup \mathcal{R}_{1}$, yielding a problem $\psi$. Then $G^{\phi}>_{W} G^{\psi}$.

Proof. (Sketched) Assume that Explode is applied to $x \in t\left[u_{1}, \ldots, u_{n}\right]_{p_{1}, \ldots, p_{n}}$ in $\phi$, the variable $x$ being replaced by $t\left[z_{1}^{\prime}, \ldots, z_{n}^{\prime}\right]_{p_{1}, \ldots, p_{n}}$, where the $z_{i}^{\prime}$ s are new variables. The resulting problem is then normalized for $\mathcal{R}_{0} \cup \mathcal{R}_{1}$, yielding the problem $\psi$. The atoms involving $x$ before Explode is applied to the atom $x \in t$ are of one of the following forms:

$-x \in t\left[u_{i}\left[z_{j}\right]_{q_{i j}}\right]_{p_{i}}$

$-v_{k} \in t^{\prime}[x]_{q_{k}}$

$-u[y]_{r} \leq x$

$-y \leq s[x]$

The last inequation is irrelevant for our measure. Note that the $p_{i} \mathrm{~s}$ are different from $\Lambda$ by the application conditions of Explode (and lemma 11), and that $x$ cannot occur in a left-hand side of an inequation. The corresponding arcs, (including a possible additional path going through $x$ and leading to an arc $\left.w^{\prime} \Longleftarrow w\right)$ are as follows:

${ }^{1}$ The alphabetic ordering is not well-founded (there are infinite descending sequences $1>0 \cdot 1>0 \cdot 0 \cdot 1 \cdots)$, but our ordering is well-founded since the first integer in a word is its length. 


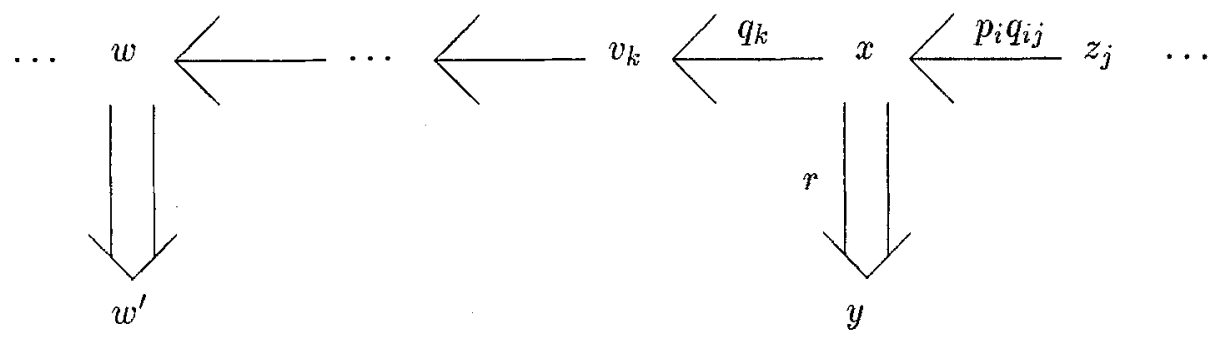

After applying Explode, the atom $x \in t$ has disappeared, and there are new atoms of the form $z_{i}^{\prime} \in u_{i}\left[z_{j}\right]_{q_{i j}}$. The atoms of the form $v_{k} \in t^{\prime}[x]_{q_{k}}$ have become $v_{k} \in t^{\prime}\left[t\left[z_{i}^{\prime}\right]_{p_{i}}\right]_{q_{k}}$. They are normalized using the rules of $\&$-Normalization into $v_{k} \in t^{\prime \prime}\left[z_{i}^{\prime}\right]_{q_{k i}^{\prime}}$ with $\left|q_{k i}^{\prime}\right| \leq\left|q_{k}\right|+\left|p_{i}\right|$. The inequation $u[y]_{r} \leq x$ has become $u[y]_{r} \leq t\left[z_{i}^{\prime}\right]_{p_{i}}$ which has to be re-normalized. This does not increase the weight since no rules (except the unification rules that will never be applied again) may introduce a variable in a left-hand side of an inequation, and the embedding rules may only decrease the depth of the variables in the left-hand sides of inequations, which decreases the first component of the weight of the paths starting with $y$, or yield an inequation of the form $u[y]_{r} \leq z_{i}^{\prime}$. In the first case, (that is if the rule $f\left(s_{1}, \ldots, s_{n}\right) \leq f\left(t_{1}, \ldots, t_{n}\right) \rightarrow \bigwedge s_{i} \leq t_{i}$ of $\mathbf{E m b}$ is applied), then the paths starting from $y$ have been replaced by paths of smaller weight (on the first component). Otherwise, the normalization yields an inequation of the form $u[y]_{r} \leq z_{i}^{\prime}$. The inequation $y \leq s[x]$ has become $y \leq s\left[\left[z_{1}^{\prime}, \ldots, z_{n}^{\prime}\right]_{p_{1}, \ldots, p_{n}}\right]$, which is still irrelevant for our measure. The graph $G^{\psi}$ is then as follows:

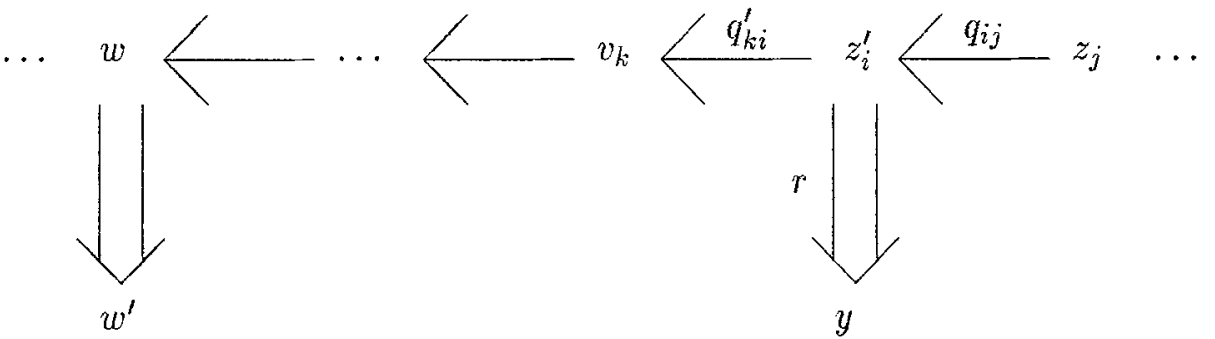

The paths of Path $\left(G^{\phi}\right)$ going to $y$, (or to some $w^{\prime}$ ) trough $x$ have been replaced by paths of the same length (and same weight in the first component) going through the $z_{i}^{\prime}$ s, but the arcs from the $z_{j}$ s to $x$ labeled with $p_{i} q_{i j}$ have been replaced by arcs from the $z_{j}$ s to the $z_{i}^{\prime}$ s labeled by $q_{i j}$ with $p_{i} \neq \Lambda$, all smaller on the second component of their weight.

We can now state our main result, following by lemmas 12 and 14 .

Theorem 15. The positive existential fragment of the first-order theory of homeomorphic embedding is decidable. 


\section{Conclusion}

We gave the first decidability result for the satisfiability of partial path orderings. We need however to go beyond:

- we wish to investigate the full existential fragment of the theory of embedding.

- we wish to investigate the full positive theory of embedding.

- we wish to investigate the extension to the positive existential fragment of any recursive path ordering. This is not straightforward, since, for example, $x \leq t$ where $t$ is ground, needs not to have only finitely many solutions.

All these works are first steps towards the study of the theory of arbitrary partial recursive path orderings (for which we recall that only the $\Sigma_{4}$ fragment is known to be undecidable). Finally, we would like to understand better the relationships with automata theory and, in particular, the combination of ordering constraints and sort constraints expressed in terms of the membership to recognizable tree languages.

\section{References}

1. Hubert Comon. Solving symbolic ordering constraints. International Journal of Foundations of Computer Science, 1(4), 1990.

2. Hubert Comon. Disunification: a survey. In Jean-Louis Lassez and Gordon Plotkin, editors, Computational Logic: Essays in Honor of Alan Robinson. MIT Press, 1991.

3. Nachum Dershowitz and Jean-Pierre Jouannaud. Rewrite systems. In J. van Leeuwen, editor, Handbook of Theoretical Computer Science, volume B, pages 243309. North-Holland, 1990.

4. Graham Higman. Ordering by divisibility in abstract algebras. Proceedings of the London Mathematical Society, 2(3):326-336, September 1952.

5. Jean-Pierre Jouannaud and Claude Kirchner. Solving equations in abstract algebras: A rule-based survey of unification. In Jean-Louis Lassez and Gordon Plotkin, editors, Computational Logic: Essays in Honor of Alan Robinson. MIT-Press, 1991.

6. Jean-Pierre Jouannaud and Mitsuhiro Okada. Satisfiability of systems of ordinal notations with the subterm property is decidable. In Proc. 18th Int. Coll. on Automata, Languages and Programming, Madrid, LNCS 510, 1991.

7. R. Nieuwenhuis and A. Rubio. Theorem proving with ordering constrained clauses. In Deepak Kapur, editor, Proc. 11th Int. Conf. on Automated Deduction, Saratoga Springs, NY, LNCS 607. Springer-Verlag, June 1992.

8. Ralf Treinen. A new method for undecidability proofs of first order theories. Tech. Report A-09/90, Universität des Saarladandes, Saarbrücken, May 1990.

9. Sauro Tulipani. Decidability of the existential theory of infinite terms with subterm relation. To appear in Information and Computation, 1991.

10. K. N. Venkataraman. Decidability of the purely existential fragment of the theory of term algebras. Journal of the ACM, 34(2):492-510, 1987. 